\title{
Sviluppo, territorio e inquinamento: il caso Gela
}

\author{
di Andrea Miccichè
}



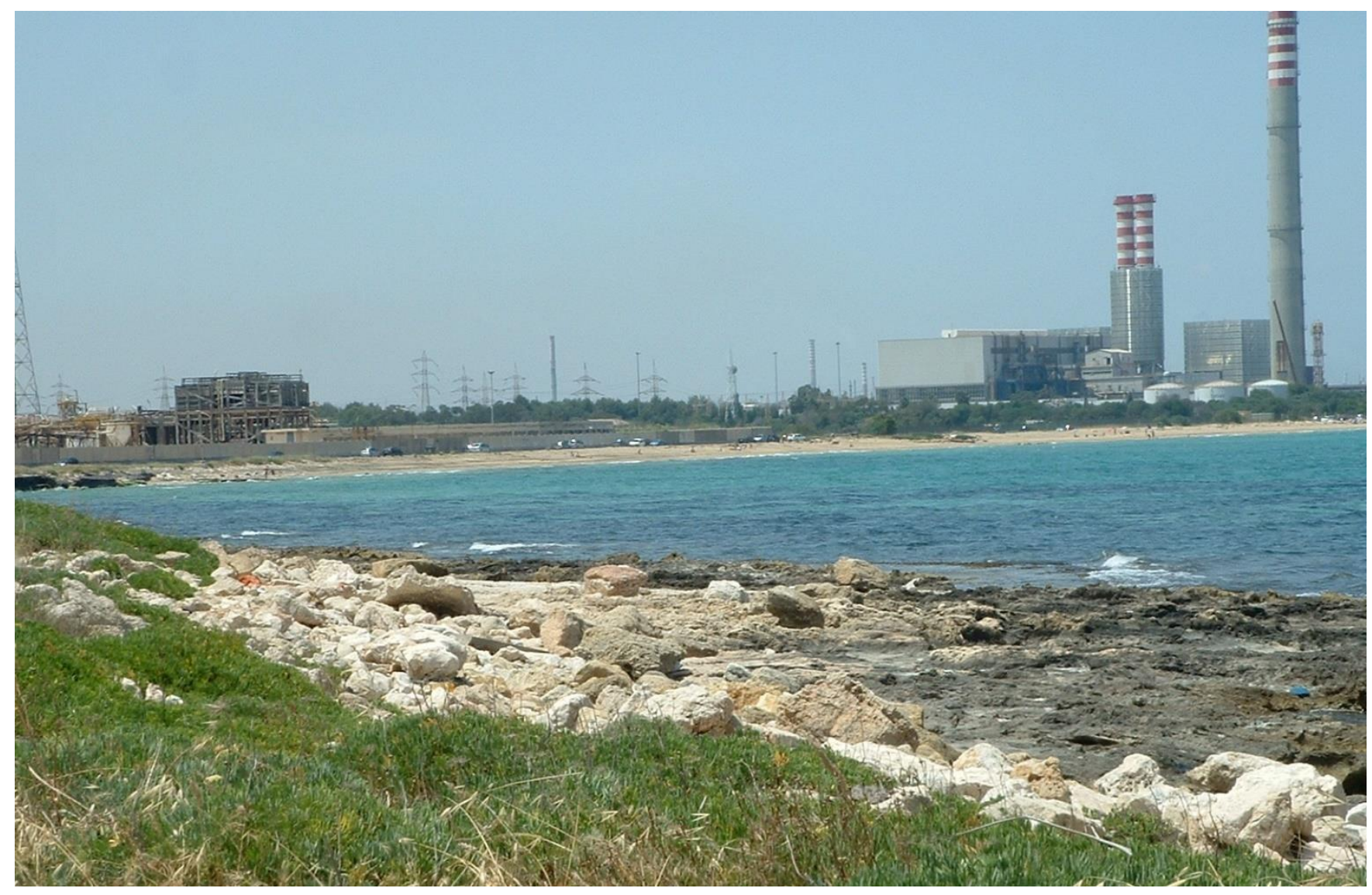

Gela - foto dell'autore

\begin{abstract}
La nascita di un polo petrolchimico dell'ENI (Ente Nazionale Idrocarburi) alle porte di Gela, nella costa meridionale della Sicilia, insieme alla scoperta del petrolio destarono nella società locale enormi speranze e un consenso generalizzato sulle potenzialità di un modello di sviluppo che avrebbe dovuto fungere da volano per la nascita e il consolidamento dell'imprenditoria siciliana. Nel corso dei decenni molte di queste aspettative sono rimaste lettera morta, lasciando però gradualmente sul tappeto sempre più interrogativi sui danni all'ambiente e sulla stessa sostenibilità economica di quelle produzioni, fino alla situazione attuale, con uno stabilimento chiuso (in attesa di riconversione) e numerosi procedimenti in corso per danni ambientali e malattie sul luogo di lavoro. Il percorso proposto permette di ricostruire fasi e contesti in cui si è svolta questa vicenda decennale, con lo scopo, anche, di favorire una riflessione sulla storia dell'ambiente come filone storiografico utile a una didattica innovativa in grado anche di mettere in relazione storie, territori e i grandi problemi del presente.
\end{abstract}




\section{Testo per docenti}

a. Per una storia dell'ambiente e per una didattica innovativa

b. Lo studio di caso

c. Una definizione

d. Una storia recente

e. Sviluppo, occupazione e inquinamento a Gela

f. Bibliografie e risorse web

\section{Testo per gli studenti}

\section{Dossier dei documenti}

3.1. Nascita di un petrolchimico

3.2. L'industria pesante a Gela tra occupazione e alterazione ambientale

\section{Le attività}

\section{Testo per i docenti}

\section{Premessa. Per una storia dell'ambiente e per una didattica innovativa}

Piero Bevilacqua qualche anno fa con L'utilità della storia. Il passato e gli altri mondi possibili [2007] si interrogava sulla crisi di una disciplina di insegnamento troppo spesso incapace di destare interesse tra gli studenti. Tra le tante cause di questa «svalutazione del passato» - alcune delle quali sistemiche, legate alla crisi degli stati nazionali, alla fine delle grandi ideologie, al "presentismo" che caratterizza il nostro tempo etc. - vi era anche una didattica troppo manualistica, incentrata sullo sforzo mnemonico, e per questo spesso dogmatica, acritica nel narrare il passato come processo "compatto", senza sfumature e contraddizioni. Un passato di cause ed effetti concatenati tra loro tale da suggerire un'idea di inevitabilità dei processi storici, dunque in aperta contraddizione con una disciplina che, invece, ambisce a ricostruire processi complessi, scelte, conflitti, progetti, idealità, dissonanze del passato. Insomma, una Storia che dovrebbe sempre essere «scienza del contesto», discutibile e sempre revisionabile, che dovrebbe alimentare una «cultura della possibilità sociale» e della cittadinanza consapevole.

Non è certamente solo un problema di manuali, spesso di ottimo livello, ma di come questi divengano la fonte per una sorta di narrazione unica e indiscutibile, sovente lontana dai problemi del presente e dalle realtà territoriali in cui sono immersi studenti e cittadini. Invece, l'insegnamento dovrebbe mettere in primo piano anche queste dimensioni - i problemi e i territori - per riconnettere l'esperienza dello studente, o più in generale del cittadino, con una Storia che sennò parrebbe sempre svolgersi in un mondo astratto e separato dall'esperienza. Rimettere insieme questi fattori significa riavvicinare la disciplina alla realtà quotidiana e farne lo strumento essenziale per comprendere i contesti e i fatti che l'hanno determinata. Dunque, non come conseguenza di processi inevitabili, ma in quanto frutto di scelte e possibilità ricostruibili e comprensibili attraverso la 
ricerca. Possibilità e scelte che si ripropongono continuamente, ci riguardano direttamente, danno valore alla partecipazione democratica e all'idea di cittadinanza, perché determinano il presente e creano le premesse per un futuro tutt'altro che inevitabile.

\section{Lo studio di caso}

Da queste premesse prende le mosse la proposta didattica sul caso Gela, centro della Sicilia Meridionale in cui è ubicato un grande complesso petrolchimico dell'ENI, esteso per circa 500 ettari. Questo, edificato agli inizi del 1960 ed entrato in funzione due anni dopo, ha dato occupazione a migliaia di persone in questi decenni, inquinando definitivamente e in maniera profonda tutta l'area circostante, ben oltre il territorio urbano. Oggi, mentre è in corso la dismissione dell'insediamento produttivo, con le conseguenti gravi ricadute occupazionali, il degrado ambientale e le abnormi incidenze di alcune patologie (malformazioni neonatali, malattie come asma, tumore ai polmoni, allo stomaco e al colon-retto ecc...) gettano una luce sinistra sulle scelte operate in un passato ormai lontano.

Da Gela, intrecciando storia ambientale e storia locale, sarà possibile ripercorrere un pezzo di storia del nostro Meridione e del nostro Paese dagli anni delle grandi trasformazioni dell' «età dell'oro» (dagli anni cinquanta al 1973) fino ai giorni nostri. Stagioni diverse della nostra storia recente in cui la presenza di produzioni industriali pesanti e il loro impatto sull'ambiente e sulle collettività circostanti, a Gela come in altre realtà similari, ha alimentato speranze e suscitato reazioni diverse: a) prima negli anni in cui il binomio industrializzazione-occupazione appariva indiscutibilmente l'unica concreta speranza per il miglioramento delle condizioni di vita delle popolazioni nelle aree arretrate e mobilitava l'opinione pubblica e i partiti politici, di governo e di opposizione, seppur con obiettivi e strategie diverse; b) poi negli anni della crisi dei poli di sviluppo e dell'industria pesante, delle contrazioni degli organici, della nuova sensibilità ecologica e dell'emergenza ambientale e sanitaria, che divenivano temi rilevanti del dibattito politico e pubblico insieme alla sempre presente questione occupazionale.

\section{Una definizione di storia ambientale}

La storia ambientale «esplora le molte dimensioni dell'interazione degli uomini con la natura attraverso il tempo». Potremmo partire da questa semplice e immediata definizione di Caroline Merchant [2002] per introdurre una branca della storiografia che si è affermata solo da pochi decenni in relazione ad una crescente sensibilità ecologica verso le sempre più evidenti e devastanti alterazioni dell'ambiente prodotte in particolare nei Paesi industrializzati negli ultimi due secoli.

L'ambiente e le sue trasformazioni sono divenuti oggetto di studio in una duplice accezione: in quanto fattore che ha influenzato e determinato le comunità umane nella loro evoluzione o come elemento che è stato radicalmente modificato dall'azione dell'uomo, soprattutto con l'avvento della società industriale, quando è cambiata la portata, l'ampiezza e la velocità dell'intervento umano sulla natura. Una ricerca e una riflessione che in questi decenni ha analizzato l'impatto dell'uomo sugli altri esseri viventi e sugli ecosistemi e che ha messo definitivamente in questione una concezione della Storia come narrazione del progresso e dello "sviluppo" dell'umanità. Quella visione ottimistica del futuro fondata sulla fiducia in un continuo e indefinito miglioramento delle condizioni di vita degli uomini, che ci appare oggi minata dai guasti irrimediabili arrecati alla nostra Terra e dalla consapevolezza della limitatezza delle risorse naturali.

\section{Una storia recente}

La storia ambientale in questi decenni, ha raccolto un'eredità di lungo periodo, in particolare quella legata alla rivista «Annales d'histoire économique et sociale» che ha avuto il merito di rivoluzionare 
i campi di ricerca e gli strumenti di indagine della storiografia aprendola al fecondo incontro con la geografia e con altre discipline sociali.

La Enviromental History a partire dagli anni settanta ha ripreso quella tradizione e quell'apertura epistemologica in un dialogo proficuo anche con la storia economica, con quella agraria e con quella urbana. Un percorso scientifico che è progredito parallelamente a una crescente sensibilità ecologica, dovuta anche a drammatici fatti di cronaca (come il disastro di Seveso del 1976, quello di Bhopal in India nel 1984 o quello di Chernobyl nella ex URSS del 1986 ecc.), che hanno sempre più acceso i riflettori sull'impatto distruttivo di una società industriale priva di controlli e di limitazioni. Un processo che in ambito scientifico è stato favorito anche dal successo di lavori come Silent Spring di Rachel Carson, che denunciava gli effetti dei pesticidi sull'agricoltura e sull'ambiente in generale e Il Cerchio da Chiudere di Barry Commoner, che lanciava un grido di allarme contro un sistema economico che aveva alterato la natura al punto da mettere a rischio la stessa riproducibilità delle risorse necessarie alla vita sulla Terra. Successi editoriali che hanno messo in questione la sostenibilità di un sistema produttivo già entrato in crisi in seguito allo shock petrolifero del 1973 e alla drammatica urgenza di una sua riconversione.

\section{Il binomio occupazione-sviluppo}

L'ambientalismo, d'altra parte, in quegli anni era entrato anche nel dibattito pubblico con la nascita di organizzazioni come il WWF (1961) o Greenpeace (1971) e successivamente con l'apparizione dei primi partiti verdi in Germania, Francia, Gran Bretagna e in Italia. Soggetti politici che introducevano nuovi temi, nuove parole d'ordine - i diritti delle generazioni future, la difesa degli animali e della biodiversità ecc. - e nuove battaglie, a cominciare dalla questione «centrali nucleari», oggetto di un referendum nel nostro Paese nel 1987 che avrebbe bandito definitivamente l'utilizzo di questa forma di energia.

Più complicato il rapporto tra ambientalismo e partiti tradizionali, anche di sinistra, nonostante 1'istituzionalizzazione della questione ambientale abbia portato all'approvazione di importanti leggi a tutela dell'ambiente o alla nascita di ministeri dell'Ambiente (in Italia nel 1986) un po' ovunque. D'altronde la questione industrializzazione come strumento privilegiato di sviluppo e di occupazione e il tema del lavoro, hanno occupato il dibattito pubblico sin dal secondo dopoguerra, acquisendo una centralità, soprattutto per sindacati e partiti di sinistra, che ha messo in secondo piano questioni rilevanti legate alla salute, allo sfruttamento delle risorse e alla tutela del territorio. Il binomio occupazione-sviluppo, soprattutto nelle aree arretrate e in particolare nel nostro Meridione è stato il motore della politica e dell'economia locale, il volto più comprensibile e popolare del nuovo corso democratico. Ha attribuito legittimità alle classi politiche locali, divenendo un elemento strutturale degli scambi politici ed elettorali tra il centro e le periferie. Gli imperativi dello sviluppo e dell'industrializzazione si sono imposti, d'altronde, in un'Italia ancora perlopiù rurale, ma immersa nel clima di grandi trasformazioni e di speranze del Miracolo economico, quando il conflitto tra esigenze della produzione industriale e le sue conseguenze in termini di inquinamento ambientale non si era ancora palesato, perlomeno in termini di dibattito pubblico. Non che non fossero già noti, nell'immediato dopoguerra i rischi legati alla produzione industriale. Ma la questione veniva ricondotta ad un problema di pianificazione urbanistica, di ubicazione degli impianti, di distanze dai centri abitati, di rapporti con le realtà produttive del territorio, ma sempre considerando il settore secondario come il principale motore dell'economia, e l'unico vero volano per lo sviluppo delle aree arretrate.

\section{L'emergere della questione ambientale}

Solo dagli anni settanta, l'urgenza della questione inquinamento (con i casi per esempio del fiume Bormida, del fiume Lambro, di Porto Marghera ecc.) ha modificato i termini del binomio 
industrializzazione-difesa del territorio e quello ancor più complicato tra occupazione e salute dei cittadini (e dei lavoratori), riequilibrando gradualmente la bilancia a favore di una maggiore attenzione agli aspetti ambientali e di lotta all'inquinamento. Un processo complesso e contraddittorio che ha destato opposizioni nelle aree maggiormente gravate da uno storico ricatto occupazionale e che non sempre politica e istituzioni sono riuscite a mediare. I casi recenti e ancora aperti dell'Ilva di Taranto e dell'ENI di Gela, tra profonde crisi aziendali e gravi situazioni di inquinamento del territorio, sono l'emblema di una lacerazione troppo spesso ancora irrisolta, nonostante l'esistenza oggi di una legislazione sull'ambiente sempre più approfondita e di una sensibilità per i temi dell'ecologia indubbiamente più matura.

\section{Sviluppo, occupazione e inquinamento a Gela}

Nel 1957 l'Ente Nazionale Idrocarburi di Enrico Mattei, fondato nel 1953, era presente in Sicilia con 12 permessi di ricerca per un totale di 367 mila ettari; aveva trovato il gas nei pressi di Catania (contrada Mendola) e un giacimento di petrolio di scarsa qualità (estremamente bituminoso) a Gela, oltre a sottoscrivere con la Regione Siciliana un importante accordo per la creazione di due società partecipate per la ricerca e l'estrazione del greggio. Un'intensificazione della presenza dell'industria di Stato nell'isola auspicata da molti settori politici imprenditoriali locali per favorire un'industrializzazione che non fosse troppo subordinata alle grandi aziende private internazionali o del Nord presenti in Sicilia, in particolare nel settore estrattifero, della raffinazione del petrolio e della chimica. Ma una presenza che era anche duramente avversata da chi riteneva, soprattutto negli ambienti confindustriali e liberali, incluse anche ampie porzioni del mondo cattolico e della DC (basti pensare a Luigi Sturzo), che l'industrializzazione della Sicilia e del resto del Meridione dovesse essere guidata dall'iniziativa privata e non dovesse comunque competere con le produzioni settentrionali.

\section{Industrializzazione siciliana}

L'industrializzazione siciliana e il ruolo dell'industria di Stato era insomma parte di un dibattito più ampio che riguardava il ruolo dello Stato e dell'impresa privata nell'economia e gli indirizzi produttivi del sistema capitalistico italiano. In Sicilia inoltre il regime di autonomia speciale in vigore dal 1947 aveva trovato la sua più importante legittimazione nel suo farsi motore dello sviluppo dell'isola. La riforma agraria e le politiche a favore della piccola proprietà contadina (in concorso con lo Stato), la legge sugli idrocarburi, la legge sull'industrializzazione e gli istituti creati per finanziare le piccole e medie imprese erano solo alcune delle importanti riforme di quegli anni, a cui si aggiungevano le tante opere pubbliche della Regione (quartieri popolari, strade, dighe, stabilimenti turistici ecc.) realizzate in concorso con la Cassa del Mezzogiorno e con lo Stato. In questi anni erano cresciuti i consumi privati passati da 292 miliardi di lire a 658 miliardi di lire, si mangiava più carne (+30\%), si spendeva di più per il vestiario (da 40 a 68 miliardi di lire), per gli alcolici (da 22 a 46 miliardi di lire), per gli spettacoli $(+122 \%)$.

Una società in movimento dunque, ma che continuava a permanere una delle aree più arretrate del paese, con una miseria che destava scandalo. Anzi proprio in quel 1957 con il I Congresso sulle iniziative nazionali e locali per la piena occupazione, organizzato dal sociologo Danilo Dolci a Palermo, la Sicilia diveniva quasi un simbolo delle ingiustizie sociali e della miseria dei tanti Sud del mondo. Questa contraddittoria transizione dell'isola verso una società modernizzata e industrializzata si svolgeva in un contesto più generale caratterizzato da una ripresa del meridionalismo - anche con la nascita della SVIMEZ (Associazione per lo Sviluppo dell'Industria nel Mezzogiorno) nel 1946 - e dall'istituzione della Cassa del Mezzogiorno nel 1950 che aveva già stanziato più di mille miliardi di lire nel suo primo decennio per la creazione di quelle dotazioni infrastrutturali ritenute necessarie alla modernizzazione dell'agricoltura e all'industrializzazione del Sud (fase della pre-industrializzazione). 


\section{La politica dei poli di sviluppo}

Con la legge di rifinanziamento 1957 (760 miliardi di lire fino al 1965), la Cassa aveva inaugurato una nuova stagione di incentivazione diretta delle imprese industriali del Sud (fase industrialista), peraltro imponendo alle aziende pubbliche di localizzare almeno il $40 \%$ dei nuovi investimenti nel Meridione. Si scommetteva su politiche di industrializzazione attiva sotto forma di incentivi fiscali, di finanziamenti a fondo perduto o a tassi di interesse agevolati, e sulla creazione di aree di sviluppo industriale che avrebbero dovuto favorire la localizzazione in determinate aree delle iniziative attraverso la predisposizione di dotazioni infrastrutturali e di servizi realizzati anche col concorso della Cassa. Un sistema di incentivi che invece di privilegiare le piccole e medie imprese, come era nel disegno iniziale, aveva finito per finanziare grandi insediamenti produttivi dell'industria di base.

Il caso dell'ANIC Gela (del gruppo ENI) era uno degli esempi di questa politica dei poli di sviluppo. La decisione dell'ENI di investire su Gela, dopo la scoperta del petrolio, la posa della prima pietra nel 1960, i lavori di costruzione con l'impiego di 7 mila operai transitati direttamente (ma provvisoriamente) dalla condizione bracciantile all'edilizia, l'inizio della produzione alla fine del 1962 generavano grandi aspettative nella popolazione gelese. L'idea che le risorse isolane venissero lavorate in loco e la promessa di un'industria chimica siciliana, rafforzata dalla scoperta del metano nel territorio ennese di Gagliano Castelferrato, nella logica della verticalizzazione del settore, del rilancio del settore zolfifero, dell'ammodernamento dell'agricoltura, attraverso la produzione di fertilizzanti a buon mercato, sembravano più che una speranza non solo per l'economia locale, ma per quella regionale.

\section{Un decollo economico mancato}

D'altra parte, secondo Eyvind Hitten e Marco Marchionni, che scrivevano nel 1970, tre differenti «interpretazioni» del processo di industrializzazione gelese coesistevano e confliggevano. Innanzitutto, una narrazione «miracolistica» dell'insediamento ENI a Gela tutta concentrata sui benefici che la comunità locale avrebbe tratto in termini di innovazione, consumi, benessere generali, miglioramento degli stili. Vi era poi una lettura «culturale» di quel processo che sottolineava il conflitto tra una realtà rurale e immobile preesistente e l'efficientismo produttivista della fabbrica moderna. Infine, vi era una narrazione «integrazionista» che salvava gli effetti positivi indotti dalla presenza del petrolchimico e attribuiva alle istituzioni locali e regionali le responsabilità del mancato sviluppo dell'area. Ma ognuna di queste narrazioni dava per scontato un decollo economico della città che gli autori contestavano radicalmente.

Il numero di lavoratori impiegati dal petrolchimico si era stabilizzato intorno alle 3 mila unità considerando l'indotto, molti dei quali provenienti da altre località isolane. E anche l'esistenza di un quartiere satellite dell'ENI edificato a Macchitella (un vero quartiere modello all'epoca), del tutto autonomo e separato da un punto di vista urbanistico e sociale dalla città era la testimonianza più evidente di una relazione articolata tra industria e comunità locale. Un rapporto complicatosi dagli anni settanta-ottanta con l'emersione della questione ambientale e con i venti di crisi a spirare sul settore della chimica. Proprio il fallimento delle aspettative che avevano accompagnato la nascita del petrolchimico, l'assenza di un tessuto industriale indotto dalla presenza dell'ENI, i danni all'agricoltura e al turismo dovuti all'inquinamento hanno reso più stridente la contraddizione tra una questione ambientale sempre più evidente e quel ricatto occupazione che negli anni ha spinto una parte della città a stringersi attorno all'unica fonte di lavoro dell'area.

\section{Tra questione ambientale e ricatto occupazionale}

Nel 2002, per esempio, la magistratura gelese ha arrestato gli impianti ritenuti fuori legge per l'utilizzo del carbone pet-coke (un sottoprodotto della distillazione dello stesso petrolio) negli 
impianti della centrale termo-elettrica, un prodotto ritenuto altamente inquinante e bisognoso di procedure particolari di smaltimento. Una decisione che ha causato la durissima protesta dei lavoratori gelesi con decine di posti di blocco e momenti di vera tensione. La mobilitazione di gran parte della classe politica locale e dei sindacati in quell'occasione, nonostante le prevedibili proteste degli ambientalisti, ha portato l'allora governo Berlusconi all'approvazione di un decreto che convertiva il pet-coke in un combustibile, risolvendo con un tratto di penna la questione[1]. Altre durissime proteste dei lavoratori si sono avute nel 2014 e poi nel 2016 sempre a difesa dei posti di lavori stavolta minacciati dai rischi di dismissione del petrolchimico per la grave crisi del sito produttivo[2]. In quest'ultima occasione si sono prolungati per una decina di giorni manifestazioni e blocchi stradali per chiedere il rispetto degli accordi sottoscritti dall'ENI con Regione e Ministero per la riconversione green dello stabilimento con la produzione di bio-carburanti e con un investimento di più di due miliardi di euro per la bonifica dell'area. Accordi che ancora oggi tardano a tramutarsi in azioni concrete[3].

\section{Un dilemma irrisolto}

In questi stessi anni, però, la procura di Gela ha avviato vari procedimenti contro l'ENI per questioni ambientali e di salute pubblica, incluse le richieste di risarcimento in sede civile per le gravissime patologie contratte degli ex dipendenti, per i casi di malformazione (sei volte superiori alla media secondo i rapporti della procura[4]) registrate nel territorio di Gela: ipospadie (una malformazione ai genitali) e tumori (soprattutto ai polmoni, al colon-retto e allo stomaco). Ultimo atto di questa vicenda è stato il rinvio a giudizio nella primavera del 2016 di 22 dirigenti dell'ENI nell'ambito di una maxi inchiesta sull'inquinamento ambientale, ancora in corso, ma che nel caso di una condanna potrebbe far presagire il definitivo disimpegno dell'azienda dal contesto gelese. La contraddizione tra occupazione e ambiente, insomma, continua a rappresentare un dilemma irrisolto: da una parte la speranza di un impegno rinnovato dell'ENI con produzioni più sostenibili dal punto di vista ambientale e con un impegno per la bonifica del territorio; dall'altra le giuste aspettative di giustizia di chi ha subito i terribili guasti ambientali e le gravi infermità che sono state conseguenza diretta (ma non facilmente dimostrabile in sede giudiziaria) di decenni di incurie e abusi ambientali.

\section{Bibliografia}

Per un inquadramento dei temi affrontati si veda:

- S. Adorno, S. Neri Serneri, Industria, ambiente e territorio. Per una storia ambientale delle aree industriali in Italia, Bologna, Il Mulino, 2009

- P. Bevilacqua, La terra è finita, Roma, Laterza, 2006

- P. Bevilacqua, L'utilità della storia. Il passato e gli altri mondi possibili, Roma, Donzelli, 2007

- G. Corona, Breve storia dell'ambiente in Italia, Bologna, Il Mulino, 2015

- S. Costantino, Il caso Gela: Industrializzazione senza sviluppo, «StrumentiRes», Rivista online della Fondazione Res, Anno V, n4, settembre, 2013

- A. De Filippo, Per una speranza affamata. Il sogno industriale in Sicilia nei documentari dell'ENI, Torino, Kaplan, 2016.

- E.Hytten, M. Marchioni, Industrializzazione senza sviluppo. Gela: una storia meridionale, Roma, Franco Angeli, 1970

- C. Merchant, Che cos'è la storia ambientale?, «Contemporanea», Vol. 5, No. 1 (gennaio 2002), pp. 135-138

- S. Mosley, Storia globale dell'ambiente, Bologna, il Mulino, 2010

- P. Saitta, L. Pellizzoni, Lo chiamavano «sviluppo»: il complicato rapporto di Gela con l'ENI, Archivio di studi urbani e regionali, n. 96, 2009. 


\section{Risorse sul web}

http://www.teche.rai.it/1956/03/viaggio-in-italia-la-sicilia-classica-e-il-petrolio/ G. Piovene, Viaggio in Italia. La Sicilia classica e il petrolio, Radio uno, 1956

http://www.raiscuola.rai.it/articoli/il-quadrilatero-industriale-del-sud-italia-19631965/6373/default.aspx

Il quadrilatero industriale del Sud Italia (1963-1965), Taranto, Ferrandina, Gela e Bagnoli, Rai Scuola, unità didattica per la scuola secondaria di secondo grado

https://www.youtube.com/watch?v=waUra-jE6JU

A. Sessa, Il cuore nero del petrolchimico di Gela, «Linkiesta», 23 febbraio 2013

https://www.youtube.com/watch?v=I3-5A6UVr9o

L. Galeazzi, D. Pierluigi, Clorosoda di Gela, il reparto killer, «Il Fatto Quotidiano», 1 agosto 2012

https://www.youtube.com/watch?v=qXyQoHTtOyo

La posa della prima pietra del futuro impianto petrolchimico a Gela, La settimana Incom del 22 giugno 1960

http://atlanteitaliano.cdca.it/conflitto/raffineria-ENI-in-localita-piana-del-signore-gela Atlante Italiano dei Conflitti Ambientali (dati, mappe, immagini del Petrolchimico di Gela)

\section{Per gli studenti: Introduzione, fonti, esercitazioni, ricerche e discussioni}

\section{Introduzione}

Che impatto hanno gli uomini sull'ambiente? Quali effetti hanno avuto e hanno le società umane sul territorio circostante?

Da alcuni decenni un certo numero di storici si è posto questi quesiti e ha cercato di raccontare le storie dei territori in cui viviamo e le trasformazioni che questi hanno subito, contribuendo ad accrescere la sensibilità nei confronti dei temi ecologici e dell'inquinamento. La storia dell'ambiente è dunque quella disciplina che ci permette di ricostruire e comprendere le scelte umane che hanno mutato e condizionato l'evoluzione degli ambienti attorno a noi, non solo a livello planetario ma anche a un livello più vicino alla nostra quotidianità.

In questo caso, per esempio, ci occuperemo della particolare vicenda di Gela, un grosso centro della Sicilia meridionale in cui è presente un importante stabilimento dell'ENI (Ente Nazionale Idrocarburi, un ente dello Stato). Questo fu costruito all'inizio degli anni Sessanta, quando si riteneva necessario l'intervento dello Stato a favore delle aree del Meridione più arretrate attraverso la costruzione di infrastrutture (strade, dighe, porti, centrali elettriche, linee ferroviarie, aeroporti, case popolari ecc.) o di insediamenti industriali.

La scelta cadde su Gela per varie ragioni (anche politiche), una di queste era stata il ritrovamento di un giacimento di petrolio che sembrava rafforzare la fattibilità di uno stabilimento in grado di lavorare in loco il greggio. In un territorio fino ad allora con un' economia agricola che offriva salari bassi e poche giornate di lavoro ai gelesi, la prospettiva di un futuro industriale destò grandi 
aspettative, confermate nei primi anni dal gran numero di operai edili 7000 (spesso ex contadini), che fu impiegata nella costruzione del petrolchimico (dal 1960 al 1963).

Le cose cambiarono negli anni successivi, quando divenne evidente che il petrolchimico non avrebbe favorito la nascita di altre aziende e avrebbe impiegato un numero limitato di lavoratori. Il rapporto tra il petrolchimico e la città divenne perciò complesso. Da una parte vi era una realtà occupazionale che andava preservata, dall'altra vi erano le speranze deluse e gli scempi al territorio che divenivano sempre più evidenti. Nel 2002, per esempio, gli stabilimenti vennero chiusi dalla procura di Gela per questioni ambientali, ma le proteste dei lavoratori gelesi e un sollecito intervento del governo risolsero il problema anteponendo valutazioni occupazionali ad altre legate alla tutela della salute pubblica. In questi anni, però, la procura di Gela ha aperto numerosi procedimenti sulla questione inquinamento e oggi gli impianti, in attesa di una riconversione green, sono fermi.

\section{Dossier di documenti}

\section{Gruppo 1. Nascita di un petrolchimico}

\section{Documento 1}

(video) V. De Seta, F. Dodi, Gela 1959: pozzi a mare, ENI, 1960, 26’20’, Archivio Nazionale Cinema d'Impresa

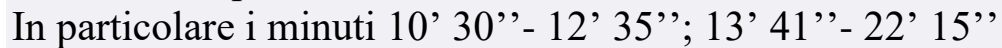

https://www.youtube.com/watch?v=UIHPI8zkVfI

\section{Documento 2}

(video) R. Sileoni, Terra di Gela, istituto Nazionale Luce, 1959

https://patrimonio.archivioluce.com/luce-web/detail/IL3000051234/1/terra-

gela.html?startPage $=0 \&$ jsonVal $=\{\% 22$ jsonVal\%22: $\{\% 22$ query $\% 22:[\% 22 \mathrm{Terra} \% 20 \mathrm{di} \% 20 \mathrm{Gela} \% 22$ $\% 22 * * \% 221, \% 22$ startDate $\% 22: \% 22 \% 22, \% 22$ endDate $\% 22: \% 22 \% 22, \% 22$ fieldDate $\% 22: \% 22$ data Normal\%22,\%22_perPage\%22:20,\%22archiveName_string\%22:\%221\%22luceFondoDocumentaril $\% 22 \% 22\}\}$

\section{Documento 3}

F. Rosso, Una ricca e imponente città petrolifera sta nascendo in Gela antica e assonnata, «La Stampa», 26 ottobre 1961.«[...] Sulla scena del petrolio siciliano, Gela è arrivata dopo Ragusa, e in un primo momento sembrò che la scoperta dell'oro nero sotto le dune sabbiose della costa, alte come colline, non avesse quel valore che i siciliani si attendevano, il petrolio, infatti, giaceva a profondità inconsueta, oltre tremila metri sottoterra, ed era di qualità scadente, addirittura bitume buono per asfaltare le strade; per estrarlo occorrevano operazioni di pompaggio eccezionali, e bisognava perfino scaldare gli oleodotti, perché il liquido pezioso appena estratto induriva come pietra. Questo accadeva nel gennaio del 1956, dopo la perforazione del primo pozzo, e molti benpensanti erano del parere che fosse meglio lasciare il bitume di Gela dove si trovava. Ma l'ENI, che ha la concessione, è un ente di diritto pubblico che deve svolgere anche una funzione sociale parallelamente all'attività economica. Inoltre, l'ing. Enrico Mattei, che lo dirige, sente il petrolio a distanze incredibili. Lo ha fiutato anche a Gela, nonostante il parere contrario dei consueti esperti, e ciò che all'inizio poteva sembrare un mezzo fallimento si sta rivelando un affare colossale; e non 
soltanto per l'economia siciliana, o di Gela in particolare. Scoperto il petrolio bisognava sfruttarlo, sia pure per farne bitume che, con lo sviluppo stradale in atto, acquista notevole valore commerciale. Si presentava però la difficoltà di trasformare il greggio: era conveniente trasportarlo allo stabilimento ENI di Ravenna, oppure creare a Gela gli impianti necessari per la lavorazione? L'ultima prospettiva era la più seducente, perché avrebbe contribuito allo sviluppo dell'industria siciliana. Fatti i calcoli a tavolino, si concluse che l'impresa, oltre a risolvere in parte le grame condizioni sociali di questo settore dell'isola, poteva essere economicamente valida. Bulldozers, ruspe, spianatrici entrarono in azione su cinquecento ettari di terra sabbiosa, livellarono dune, aprirono canali, tracciarono strade. Il 19 giugno del 1960 era nato il più colossale impianto petrolchimico d'Italia, forse d'Europa. E' trascorso poco più d'un anno e dov'era la deserta e sterile altalena di dune, già si profila lo schema della nuova città petroliera; le alte ciminiere della centrale termoelettrica trafiggono le nuvole di sabbia, il magazzino per i fertilizzanti incurva le volte delle sei navate per inserirsi armoniosamente nel paesaggio ondulante, la geometrica architettura dello stabilimento per le materie plastiche è quasi rifinita, gli aerei geroglifici della raffineria incominciano a delinearsi, la banchina del porto già s'avventura tra i marosi.[...] Nei campi, tra $\mathrm{i}$ pozzi da cui scaturisce la preziosa energia liquida, squadre di contadini si piegavano in gesti ritmici a cogliere i boccoli del cotone. Nessuna immagine potrebbe convenientemente descrivere il contrasto fra la dimensione avveniristica in cui si muove la città del petrolio e il mondo di quei contadini quasi fossilizzati in un'esistenza primordiale. Il trauma è stato troppo violento perché essi possano rendersene conto, la frattura tra la vita di ieri e quella di oggi è troppo profonda perché essi possano accettare subito il nuovo ordine. Dal 19 giugno 1960, cioè da quando l'ENI ha incominciato i lavori per il grandioso complesso petrolchimico, ad oggi è trascorso meno di un anno e mezzo, una parentesi di tempo troppo breve per incidere su esistenze che si ripetono uguali da secoli; eppure qualche cosa è mutato anche a Gela. Lo si avverte parlando con gente di città e contadini, soprattutto con questi ultimi.[...] Le imprese che costruiscono gli stabilimenti petrolchimici per conto dell'ENI occupano attualmente circa quattromila operai, in gran parte manovali, che sono contadini evasi dai campi. Fino all'anno scorso guadagnavano circa settecento lire al giorno; lavorando per la costruzione dello stabilimento petrolchimico guadagnano 59 mila lire al mese, oltre alle garanzie contrattuali. Rarefacendosi nelle campagne la mano d'opera, la richiesta è aumentata e anche le condizioni dei contadini rimasti sono migliorate sensibilmente. Tuttavia, questo aspetto positivo non può essere considerato determinante: il previsto assorbimento di cinquemila operai entro l'ottobre del 1962, data in cui il complesso petrolchimico incomincerà il ciclo di produzione, non basterebbe a segnare la frattura definitiva fra i due mondi in contrasto, se altri elementi non interverranno ad allargare la breccia aperta nell'immobilismo sociale dalle iniziative dell'ENI[...].» 


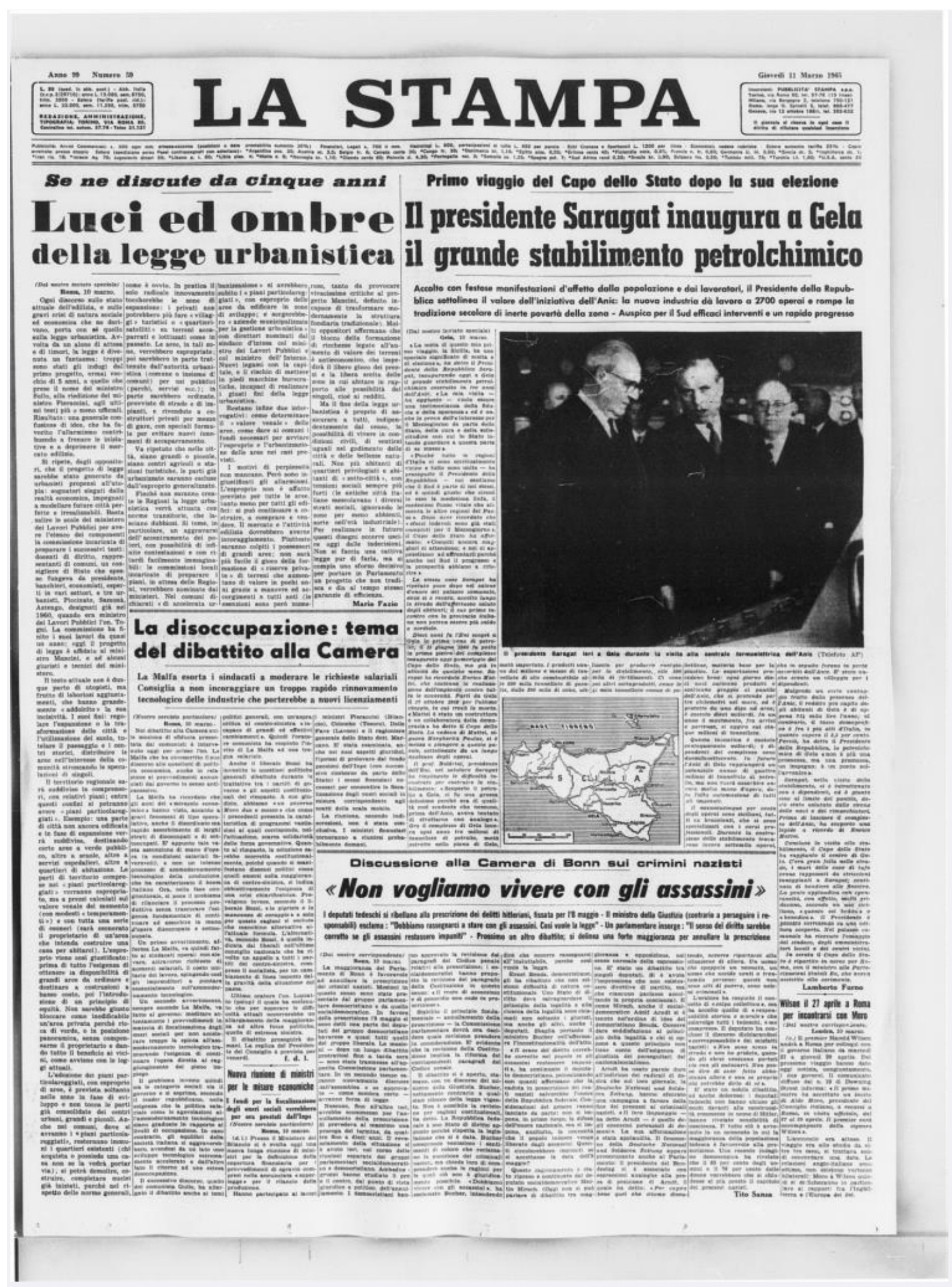

Documento 4

L. Furno, Il Presidente Saragat inaugura a Gela il grande stabilimento petrolchimico, «La Stampa», 11 marzo 1965. 
«La meta di questo mio primo viaggio, la Sicilia, ha uno speciale significato di scelta e di elezione», ha detto il Presidente della Repubblica Saragat, inaugurando oggi a Gela il grande stabilimento petrolchimico costruito in tre anni dall'Anic. «La mia visita - ha aggiunto - vuole essere una testimonianza della fiducia e della speranza» ed è anche la prova dell'interesse per il Mezzogiorno da parte dello Stato, della cura e della sollecitudine con cui lo Stato intende guardare a questa parte di se stesso». «Poiché tutte le regioni d'Italia ci sono spiritualmente vicine e tutte sono unite - ha proseguito il Presidente della Repubblica — noi sentiamo che il Sud è parte di noi stessi, ed è quindi giusto che circoli in esso la medesima linfa, il medesimo flusso vitale che alimenta le altre regioni del Paese». [...] Il prof. Boldrini, presidente dell'ENI, nel salutare Saragat ha riepilogato le difficoltà incontrate per costruire lo stabilimento: «Scoperto il petrolio a Gela, ci fu una grossa delusione perché era di qualità cosi scadente che nessuno, prima dell'Anic, aveva tentato di sfruttarne uno analogo». Ora il complesso di Gela lavora ogni anno tre milioni di tonnellate di petrolio, metà estratto nella piana di Gela, metà importato. I prodotti vanno dal milione e mezzo di tonnellate di olio combustibile alle 690 mila tonnellate di gasolio, dalle 390 mila di coke, utilizzato per produrre energia per lo stabilimento, alle 500 mila di fertilizzanti. Ci sono poi altri sottoprodotti, come le 1,5 mila tonnellate annue di polietilene, materia base per la plastica. Le esportazioni procedono bene: ogni giorno dieci navi caricano prodotti e scaricano greggio al pontile dell'Anic, che si protende per tre chilometri nel mare, ed è protetto da una diga ad arco; è costato dieci miliardi. In un anno il movimento, fra arrivi e partenze, si aggira sui cinque milioni di tonnellate. Questa iniziativa è costata centoquaranta miliardi; i dipendenti del complesso sono duemilasettecento. In futuro l'Anic di Gela raggiungerà un potenziale annuo di quattro milioni di tonnellate di petrolio, ma non potrà assorbire ancora molta mano d'opera, data l'alta automazione di tutti gli impianti.[...] Malgrado un certo vantaggio tratto dalla presenza dell'Ente, il reddito pro capite degli abitanti di Gela è di appena 814 mila lire l'anno; al contrario, il tasso demografico è fra i più alti d'Italia, in quanto supera il 3,5 per cento. Perciò, ha detto il Presidente della Repubblica, la petrolchimica di Gela «non è più una promessa, ma una premessa, un impegno; è un ponte sull'avvenire». 




Gruppo 2. L'industria pesante a Gela tra occupazione e alterazione ambientale 
E Hytten, M. Marchionni, Industrializzazione senza sviluppo. Gela: una storia meridionale, Franco Angeli, 1970, pp. 48-49

«[...] è inutile sottolineare che la grande importanza nazionale e internazionale dell'impianto petrolchimico di Gela non comporta automaticamente una simile importanza livello regionale e locale. [...] Si è già detto della mancanza di effetti moltiplicatori in termini puramente economici dell'impianto gelese, mancanza in gran parte attribuibile alla stessa natura tecnologica dell'ANIC e alle caratteristiche del gruppo industriale di cui fa parte. Un discorso abbastanza simile vale per l'elemento che rappresenta l'effetto economico più diretto, il monte salari erogato dalla fabbrica. [...] È fuor di dubbio che l'isolamento di gran parte delle maestranze nel villaggio residenziale praticamente autosufficiente [il quartiere ENI di Macchitella nda] [....], la quasi totale assenza di occasioni di investimento produttivo ed altri fattori strutturali ancora, fanno sì che l'ammontare totale dei salari non dia alcuna indicazione sicura sul capitale effettivamente assorbito dall'economia della zona. Inoltre il successivo rialzo dei prezzi in seguito all'aumentata domanda creatasi negli ultimi anni contribuisce a neutralizzare gli effetti positivi, per la comunità nel suo insieme, che l'afflusso di capitali in alcuni settori dell'economia avrebbe potuto avere. Il presupposto di un radicale cambiamento dell'assetto economico locale, come base per la domanda del perché non ne siano seguiti degli effetti di rinnovamento anche sociale, ci sembra quindi mal posta e tale da sviare il discorso dai termini reali del problema. Non si vuole certo affermare [...] che non ci sia stato un cambiamento economico di nessun genere [...]. Ma questo cambiamento [...] risulterà sicuramente, all'analisi più accurata, molto meno significante di quanto le cifre globali darebbero a intendere.

\section{Documento 2}

\section{F. Amabile, Un decreto del governo riapre Gela, «La Stampa», 8 marzo 2002}

Via libera del governo al decreto che autorizza l'utilizzo del pet coke negli impianti di combustione dell' Agip di Gela. Il provvedimento consente di scongiurare la chiusura degli impianti del petrolchimico della città (dove ieri in occasione di un nuovo sciopero generale sono scese in piazza 30 mila persone) e l'impasse che si era creata nei giorni scorsi, rimuovendo la causa che aveva provocato il sequestro di alcuni impianti della Raffineria Agip da parte della magistratura, ma non le proteste degli ambientalisti che continuano a considerarlo «una bomba ambientale».

[...] «Una soluzione positiva anche se non ideale», l'ha definita il ministro dell'Ambiente Altero Matteoli. «Avrei preferito una soluzione che non fosse dettata dall'emergenza - ha spiegato Comunque sono stato rassicurato dal fatto che i problemi del petrolchimico di Gela non derivano da rilievi ambientali ma dal formalismo della normativa». Il ministro ha anche ricordato di aver stanziato nel '94 dei fondi per il risanamento che però non sono ancora stati utilizzati. Adesso i fondi sono nuovamente disponibili e, ha concluso Matteoli, «si dovrà quanto prima avviare il processo di risanamento complessivo». Il decreto, poche righe in tutto, stabilisce che per gli impianti di combustione con potenza termica nominale per singolo focolare, superiore ai $50 \mathrm{Mw}$, è consentito l'uso di coke da petrolio con un contenuto di zolfo non superiore al 3\% di massa.

«È una truffa, un nuovo condono - ha denunciato il presidente dei Verdi Alfonso Pecoraro Scanio. Invece di stanziare fondi per salvaguardare salute e occupazione il governo ha preferito legalizzare inquinamento e malattie. Il decreto classifica il pet-coke come un combustibile, nonostante si tratta in realtà di un residuo del processo di raffinazione del petrolio, e dunque di una sostanza altamente dannosa. Non solo viene by-passata la legge Ronchi ma soprattutto viene messo a rischio la salute di chi lavora e vive a Gela. Il petrolchimico resta così uno dei siti a più alto rischio una vera bomba ambientale che il governo Berlusconi preferisce mantenere». 
Viaggio nel nuovo Texas del Petrolchimico, «La Stampa», 8 marzo 2002

«[...] Anche in questi giorni difficili Gela sembra quella di sempre: precaria e indefinita. Ma anche incazzata, disillusa, delusa nel vedere sbiadire un sogno mai avverato ma oggi seriamente compromesso da una parola estranea, importata ma immediatamente entrata nell'uso quotidiano per il suo elevato valore intimidatorio. Stiamo parlando del pet-coke, che i capi della raffineria (ma anche gran parte degli operai minacciati dalla disoccupazione) definiscono carburante buono per alimentare le centrali e il ciclo produttivo e, invece, i magistrati considerano scorie altamente nocive per la salute. Certo, la storia è vecchia perché da anni Gela convive coi veleni, ma adesso c'è di nuovo che i magistrati non intendono più fingere di non vedere e di non sapere. Già, chi poteva immaginare - quarant'anni fa - che una parte del sogno del riscatto sodale siciliano, il mito del «nuovo Texas» di Enrico Mattei, sarebbe diventato questo terribile boomerang di oggi.

[...] Storia crudele, quella cominciata alla fine degli Anni Cinquanta. A Gela vivevano poco più di 35.000 persone. Ricorda l'operaio Antonio La Falaga, uno dei «vecchi» del Petrolchimico: «C'era tanta povertà tra i lavoratori. I proprietari stavano bene con la produzione del cotone e del grano, $\mathrm{i}$ braccianti dovevano sottostare alla vergogna dell'arruolamento giornaliero in piazza. La riforma agraria apriva i primi spiragli e la terra parcellizzata dava della buona uva precoce che veniva esportata». E poi c'era il mare. «Il più bel mare del mondo» enfatizza l'operaio, spalleggiato dal collega Antonio Scordio. Entrambi sembrano rimpiangere il tempo di quando Gela viveva anche col pesce e la costa era una spiaggia continua. Sabbia chiara e fine, «la sabbia dorata» celebrata da Quasimodo nella poesia «Ad un soldato nemico», recitano i due operai. Tutto ciò non bastava, però, a frenare l'emorragia che portava linfa lavorativa al Nord e nei freddi paesi dell'Europa: un crudele e disumano cammino della speranza, alla ricerca di una vita migliore. Non è difficile, dunque, comprendere quali aspettative potesse generare la scoperta del petrolio e i discorsi di un uomo come Enrico Mattei, capace di accendere entusiasmi e di accattivarsi le simpatie popolari [...].

La metamorfosi è repentina. La popolazione raddoppia, arrivano i tecnici da Ravenna e dal Nord, nasce una città nella città assolutamente autonoma ed autosufficiente. Il «quartiere petrolifero» di Macchitella con le sue casette linde e ordinate, i suoi negozi, gli uffici, persino un distaccamento dei vigili urbani. Una razionalità che avrebbe dovuto far da guida alla prevedibile espansione, figlia del benessere prodotto dai quasi cinquemila stipendi della raffineria e delle rimesse degli emigrati.

Soldi incanalati soprattutto nell'acquisizione del «mattone»: la casa per sé, per i figli e per i figli dei figli. Ventimila vani abusivi, la crescita nel caos, [...] L'Iacp che costruisce le case popolari a mare, a due passi dalla spiaggia dove forse si poteva pensare ad organizzare il turismo. $\mathrm{O}$ forse no, dal momento che il Petrolchimico - non era ancora tempo di depuratori, marmitte catalitiche e salvaguardia dell'ambiente - aveva fatto il vuoto col puzzo di uova marce e periodici divieti di balneazione. Così Gela si buscò il marchio, ancora non dismesso, di «Gela la brutta». La speranza iniziale si andava trasformando - quale che fosse il partito al potere - in una strenua difesa del presente anche se orrido ma a stipendio fisso. L'alternativa, d'altra parte, sarebbe stata di arrivare vent'anni prima alla considerevole cifra dei 16.000 disoccupati di oggi, anche se c'è più d'uno che 
su questi dati non si giocherebbe la faccia [...]. ».



Documento 4

(video) G. Pipitone, S. Bellotti, Clorosoda di Gela, il reparto killer, Il Fatto Quotidiano 


\section{Le attività}

\section{Il gioco di comitato}

La classe si dividerà in 4 gruppi che analizzeranno i due blocchi di fonti per 20-30 minuti circa. A questo punto ciascun gruppo riceverà una carta tra 4 possibili: a) accusa; b) difesa; c) giudici; d) giuria.

1. L'accusa dovrà assumere il punto di vista del "tempo presente" mettendo in evidenza le criticità del petrolchimico, il suo impatto sul territorio, i rischi ambientali e sanitari. Ogni affermazione dovrà comunque fondarsi sulle fonti proposte e sulle informazioni in esse contenute (del blocco $1 \mathrm{o} 2$ ). L'accusa formulerà 3 affermazioni rivolte alla difesa. Queste affermazioni andranno argomentate oralmente nel corso del dibattito.

2. La difesa, assumerà il punto di vista del "tempo passato" con l'obiettivo di evidenziare motivazioni, speranze, opportunità che hanno accompagnato la nascita del polo petrolchimico di Gela. Nello specifico, si dovrà rispondere alle 3 affermazioni rivolte dall'accusa con altrettante affermazioni, i cui contenuti dovranno derivare necessariamente dalle fonti proposte. Queste affermazioni andranno argomentate oralmente nel corso del dibattito.

3. I giudici entreranno in gioco in un secondo momento ed esprimeranno il punto di vista dei "diversi contesti storici". Ovvero dovranno analizzare le affermazioni dell'accusa e della difesa e proveranno a formulare tre sentenze che tengano conto degli argomenti di entrambi. Queste affermazioni dovranno comunque fondarsi sulle fonti proposte oltre che sulle affermazioni formulate dai compagni. Queste affermazioni andranno argomentate oralmente nel corso del dibattito.

4. La giuria, valuterà la validità delle 9 affermazioni, ne vaglierà la fondatezza rispetto alle fonti proposte (eventuali affermazioni non sufficientemente fondate sulle fonti verranno ricusate) e a maggioranza dei suoi componenti deciderà il vincitore sulla base di una sentenza finale che giustifichi la scelta.

5. I gruppi, in conclusione, discuteranno e definiranno modalità e contenuti per una presentazione Power Point o Prezi che descriva lo svolgimento delle attività e le sue conclusioni.

\section{Una possibile attività conclusiva. Oltre la Storia, le scelte del passato e le sue conseguenze possibili}

Nella prima metà degli anni settanta lo stabilimento petrolchimico dell' ISAB venne costruito nella zona di Marina di Melilli (immagine in basso) nel siracusano. La località, dove già erano presenti altri insediamenti industriali, venne preferita ad altri due possibili siti: San Vito Lo Capo (nel trapanese, immagine a sinistra) e la zona di Vindicari (tra Noto e Pachino, immagine a destra), riserva naturale dal 1984[5]. Oggi queste due zone sono poli di attrazione turistica di importanza nazionale e veri tesori naturalistici.

Immagina se la localizzazione dell'ISAB fosse stata, non a Marina di Melilli, ma in una delle altre due località. 
1. Cerca informazioni su una delle due località (web, biblioteca, emeroteca ecc.) e scrivi un ipotetico reportage controfattuale di denuncia in cui descrivi l'inquinamento e l'alterazione dell'ambiente dovuti alla produzione industriale



Note:

[1] F. Amabile, Un decreto del Governo riapre Gela, «La Stampa» dell’8 marzo 2002

[2] ENI, corteo a Gela contro la minaccia di chiusura del petrolchimico. Domani lo sciopero generale nel gruppo, «L'Huffington Post» del 28 luglio 2014; G. Pipitone, Gela, sciopero generale della città. In diecimila marciano per l'accordo di riconversione del petrolchimico ENI, «Il Fatto Quotidiano.it» del 26 gennaio 2016.

[3] La riconversione della raffineria, convocato un nuovo vertice al ministero: sciopero nel settore coibenti, «Quotidiano di Gela» del 7 aprile 2017

[4] L. Tondo, Gela, inchiesta della Procura sui bambini malformati, «La Repubblica.it» del 27 febbraio 2017.

[5] S. Adorno, L'area industriale siracusana e la crisi ambientale degli anni settanta, in S. Adorno, S. Neri Serneri, Industria ambiente e territorio. Per una storia ambientale delle aree industriali in Italia, Il Mulino, Bologna, 2009, p. 297. 
